\title{
Observations \\ sur les rides sous-marines du plateau continental *
}

\author{
PAR
}

\author{
G.-A. Auffret \\ Centre Océanologique de Bretagne, Brest
}

\author{
L. Berthois \\ Rennes
}

ET

\author{
J.-L. Douville \\ C.N.R.S., Station biologique, Roscoff
}

\section{Introduction}

L'intérêt économique croissant, lié aux zones relativement peu profondes du plateau continental, explique le regain d'attention porté aux processus sédimentaires dont cette zone est le siège.

Ces processus sont en effet à la fois bénéfiques. puisquils contribuent à la constitution d'accumulations de matériaux sédimentaires exploitables, mais ils peuvent également constituer une gêne pour l'implantation de structures fixes sur le fond marin, ou le creusement de chenaux pour la navigation. Ces faibles profondeurs ont été tout naturellement les premières étudiées par les océanographes et les géologues (Pruvost, 1897; Thoulet, 1912; Dangeard, 1923), mais curieusement, au fur et à mesure du développement des techniques, leur étude a été relativement délaissée pour celle des grandes profondeurs, et beaucoup reste à apprendre sur ce domaine.

\section{Morphologies et hydrodynamismes}

La figure I situe les différentes régions étudiées en détails, sur lesquelles nous nous appuierons pour illustrer notre exposé.

Les accumulations sédimentaires, présentes en Manche et en mer Celtique, peuvent être classées selon une hiérarchie de forme depuis les grands corps sableux, tels ceux qui caractérisent les fonds du Pas-de-Calais ou du banc de

\footnotetext{
* Contribution n" 385 du Départemen Scientifique, Centre Océanologi-
} que de Bretagne, B.P. 337, 29273 Brest (France). la Chapelle, jusqu'aux minces rubans sableux présents au sud de la Manche Occidentale.

\section{A) EST DE LA MANCHE ORIENTALE : LA BASSURELLE}

Les grands corps sableux, tels ceux de la Bassurelle (fig. 2), ont leur axe principal parallèle au grand axe de l'ellipse des courants de marée; leurs flancs sont festonnés de grandes dunes hydrauliques dont la crête est également parallèle à la direction générale (fig. 3). Un traict de troïka a été réalisé suivant le profil $X-Y$ (fig. 2 et 3 ). Tous les creux des rides sont occupés par une faune fixée de Polychètes sédentaires (Lanice conchilega). Dans la partie sud du tracé, les Polychètes cèdent la place à des Alcyonnaires. En outre, de gros débris coquilliers de Lamellibranches parsèment quelques aires déprimées. Enfin, les crêtes des grandes dunes hydrauliques sont occupées par des systèmes de petites rides ayant de 0,15 à $0,20 \mathrm{~m}$ de longueur d'onde et 0,02 à $0,05 \mathrm{~m}$ d'amplitude. Ces petites rides sont actives, car les photographies sous-marines permettent fréquemment d'apercevoir un petit bourrelet sableux sur la pente abrupte, signalant le déplacement du sable. La présence de Lanice conchilega, dont la durée de vie est de deux ans à deux ans et demi (P. Hommeril, 1967), implique une assez grande stabilité des fonds sableux pendant une longue période. Une érosion d'une quinzaine de centimètres entrainerait le déracinement de l'animal. Un remblaiement de $10 \mathrm{~cm}$ provoquerait un enfouissement total. Mais, dans leur zone d'occurrence, les Polychètes ont tous des hauteurs identiques au-dessus du sable. On peut donc en inférer qu'ils ont atteint le même stade de développement. Ceci suggère qu'ils ont pu commencer à croître au même moment, peut-être à la suite d'importants mouvements sédimentaires ayant entrainé un bouleversement de la bathymétrie. Un tel bouleversement aurait pu être pro- 


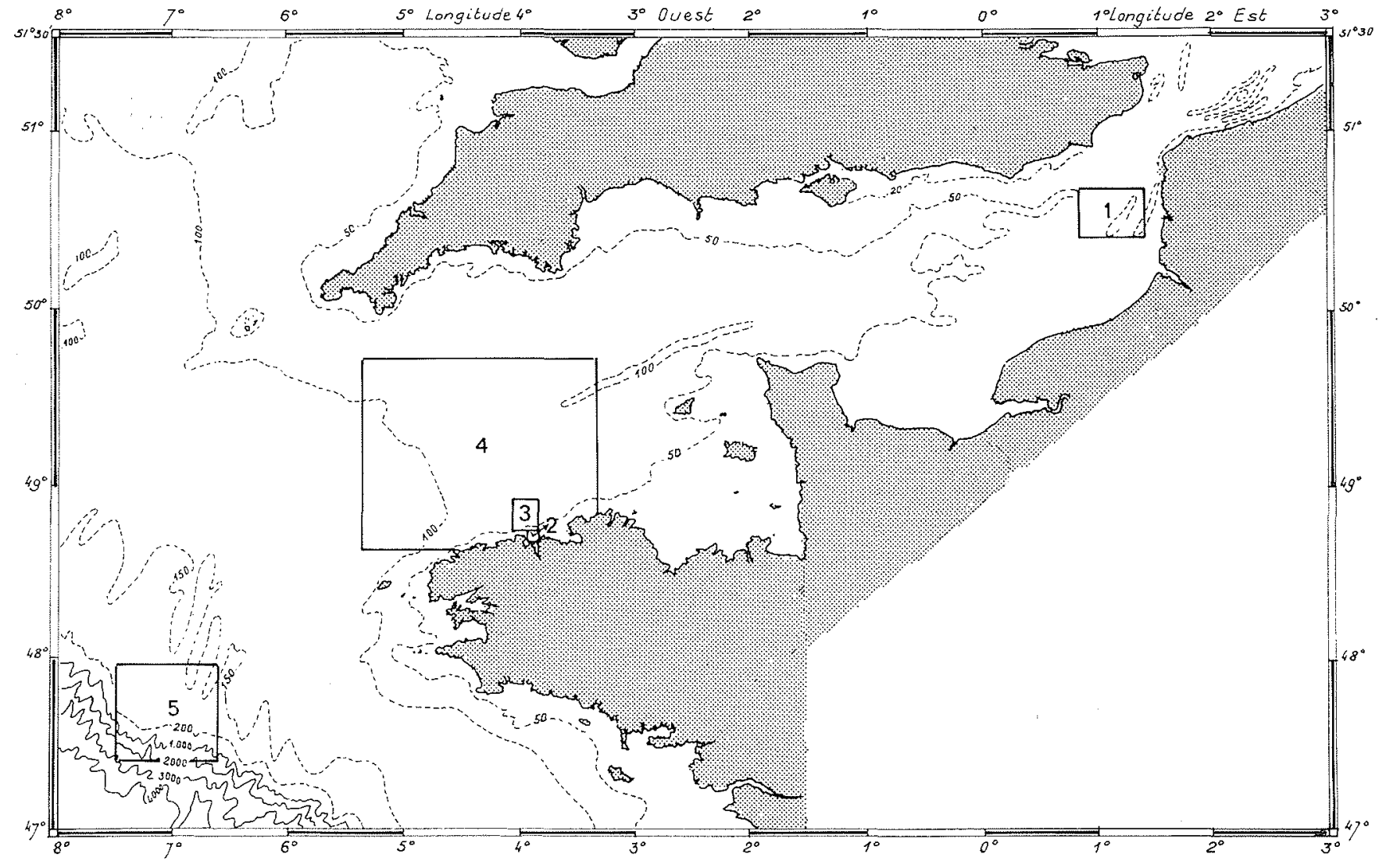

1 / Situation des différentes zones étudiées.

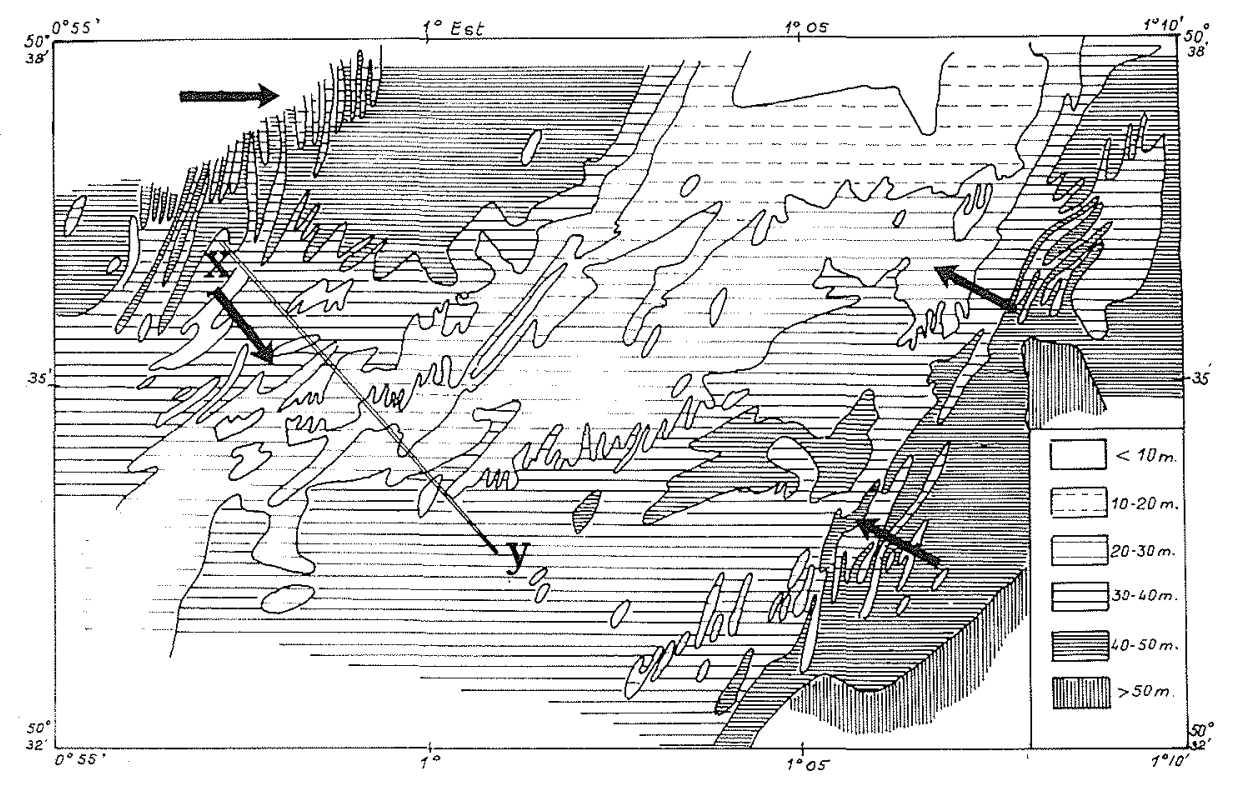

2 / Carte schématique de la Bassurelle,

$X Y$ : traict de troika, les flèches noires Indlquent les sens du transport d'après la morphologle des dunes. 
voqué par la conjonction de tempête el de marée à fort coefficient offrant les conditions optima de déplacement des matériaux.

Les mesures de courants, que nous avons réalisées dans cette zone, ont montré qu'ils sont influencés dans une large mesure par les reliefs sous-marins. Ils s'annulent à l'abri d'un haut fond ou sont guidés dans les inter-rides. mais, de façon générale, l'évolution s'apparente au cas idéal d'une onde progressive : vitesse maximale aux étales de montant (flot) et perdant (jusant). Le courant traversier. portant vers l'ouest-nord-ouest, semble avoir une influence prédominante à l'est de la zone sur l'orientation des crêtes des dunes hydrauliques qui lui sont perpendiculaires. Son importance apparait clairement sur un diagramme exprimant pour les différentes directions le nombre de "kilomètres d'eau "s’étant écoulé au cours du cycle de marée. A l'ouest, la situation est plus complexe. La crête des rides NS par les fonds de 40 à $50 \mathrm{~m}$ s'oriente encore perpendiculairement au courant traversier lorsque la profondeur diminue. Dans les conditions ordinaires, nos mesures ont montré que la vitesse moyenne au voisinage du fond était de lordre de $15 \mathrm{~cm} / \mathrm{s}$ et le maximum de $42 \mathrm{~cm} / \mathrm{s}$. L'étude de la distribution granulométrique des sédiments prélevés (fig. 4, zone 1) montre que cetle vitesse est suffisante pour assurer leur mise en suspension ainsi que leur transport.

\section{B) SUD DE LA MANCHE OCCIDENTALE}

Au sud de la Manche Occidentale, les accumulations sableuses sont présentes à trois niveaux : au premier niveau. vers $-25 \mathrm{~m}$ (Pierre Noire), sous le zéro marin. on trouve une accumulation de sable fin (en surface) épaisse au maximum d'une dizaine de mètres. Au deuxième niveau. vers - $60 \mathrm{~m}$ (Trezen Vraz), on trouve dune part une nappe de sable hétérogène grossier épaisse au maximum de cinq mètres, sur laquelle sont superposées localement des dunes hydrauliques constituées de sables fins, épaisses au maximum d'une dizaine de mètres. Enfin. plus au large. sur la plateforme calcaire, on trouve des accumulations sableuses isolées sous forme de dunes hydrauliques qui affectent souvent la forme de "barkhanes".

Le banc de la Pierre Noire est limité au nord par le haut fond du Rater et au sud par les roches de la Pierre Noire qui le séparent de la zone "estuairienne" de la baie de Morlaix. Sa profondeur moyenne est de $15 \mathrm{~m}$ environ audessous du niveau des plus basses mers: il s'allonge sur $2.4 \mathrm{~km}$ dans le sens est-ouest.

Nous avons dressé une carte bathymétrique de la zone (fig. 5) à partir de nos levés et des données de la carte marine. Sa morphologie est complexe. Le socle paléozoïque et prótérozoïque constitue le substratum des côtes rocheuses et des nombreux ilots de la baie. Un profond chenal en partie colmaté correspond a rancien cours de la Penzé el de la rivière de Morlaix.

Huit points ( $U, V, X, Y, W, Z, 7028,7029$ ) ont fait lobjet de mesures dans et autour de la zone étudiée. Seul le point 7029 n'a pu donner de résultats qu'en vive-eau: les sept autres points sont caractérisés par leurs roses de courant en vive-eau et en morte-eau tracées a partir de mesures réalisées sur plusieurs cycles de marées. Nous avons calculé. à partir des médianes relevées sur les courbes granulométriques (fig. 4), le diamètre sédimentologique el déduit de labaque de Bonnefille (fig. 8) la vitesse critique de frottement caractéristique des stations de mesure de courant. En appliquant la formule de Sternberg. nous en avons déduit la vitesse critique $V_{a}, 100 \mathrm{~cm}$ audessus du fond. Sa valeur est de l'ordre de $20 \mathrm{~cm} / \mathrm{s}$ dans lensemble de la zone. sauf au point $U$ où elle est légèrement supérieure. Pour les points $Z, W, 7028$ et 7029, ou nous niavions pas d'indications sur la granulométrie du sédiment. nous avons supposé une vitesse critique de $20 \mathrm{~cm} / \mathrm{s}$. L intégrale de la courbe $V_{u}$ par rapport à $t, V_{u}$ étant borné inférieurement par $V$. représente la distance curviligne qui serait parcourue par une molécule d'eau restant soumise dans le temps et l'espace aux conditions de la rose des courants du point en question. Les résultats de ce calcul, en grandeur et en direction, sont figurés sur la figure 5. L'ordre de grandeur de la résultante, par rappont aux composantes dont elle est la somme. est souvent faible

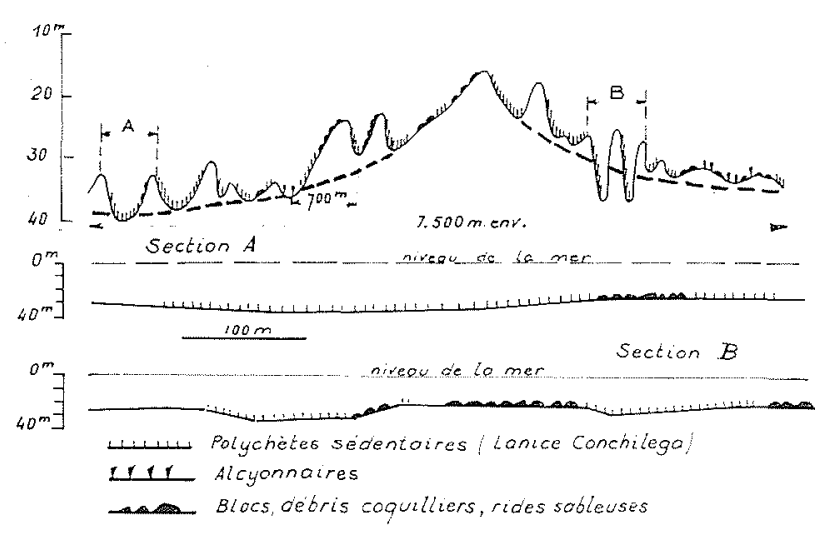

3 / Profil bathymétrlque le long du tralct de troïka $X Y$.

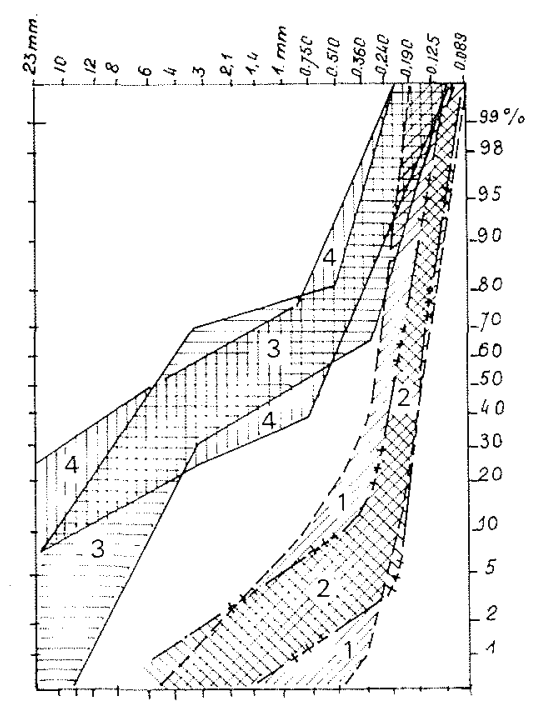

4 / Faciès granulométriques, observables dans les différentes zones. 


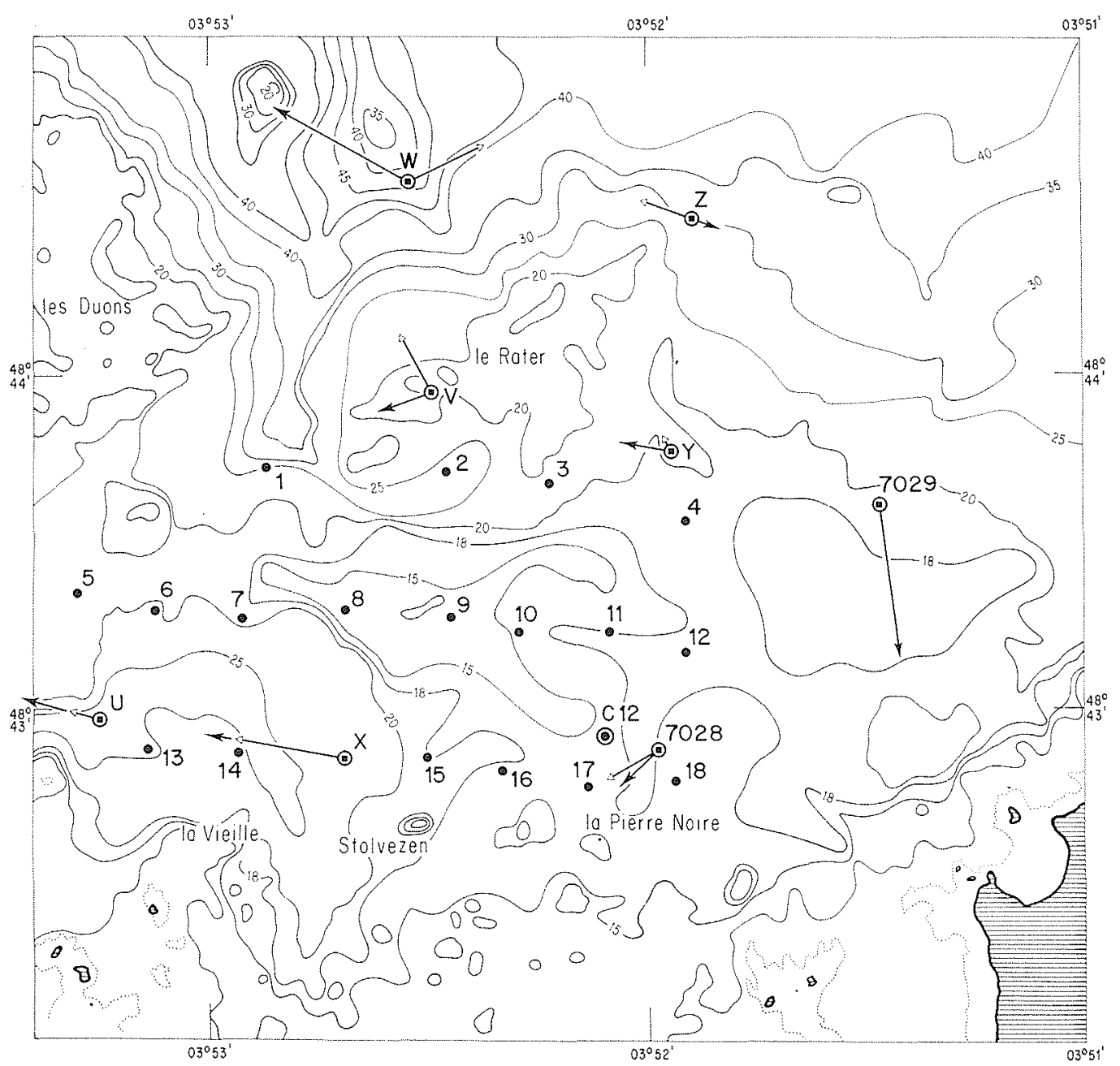

5 / Carte bathymétrique de la zone étudlée. Les prélèvements numérotés de 1 à 18 sont indiqués par des polnts noirs; les polnts de mesures courantographlques par un carré noir; les résultantes des courants de fond sont représentées à l'échelle de la carte bathymétrique en vive-eau par une flèche noire, et en morte-eau par une flèche claire.

et, de ce fait, ces indications doivent être considérées avec prudence. Néanmoins cinq stations entourant le banc de la Pierre Noire $(U, X, 7028, Y, V)$ sont caractérisées par une résultante dirigée vers louest. Au nord-est du banc (point 7029), en vive-eau, une nette dominance sud-est est observable.

La baie de Morlaix est exposée aux houles du secteur nord. En l'absence de tout matériel de mesure adapté, nous avons établi deux plans de vagues de la zone pour. tenter une approche théorique de ce problème. Les conditions adoptées sont les suivantes: vent d'ouest-nordouest $\left(\lambda_{0}=100 \mathrm{~m}, T_{0}=12 \mathrm{~s}, 2 a_{0}=6 \mathrm{~m}\right)$; vent d'est- nord-est, $\left(\lambda_{0}=60 \mathrm{~m}, T_{0}=6,2 \mathrm{~s}, 2 a_{0}=2 \mathrm{~m}\right)$. En toute rigueur, les résultats devraient être "calés" par une vérification expérimentale, mais il apparait, d'ores et déjà, que deux zones de haute énergie peuvent être distinguées, la première par vent d'est - nord-est, centrée sur le nord de l'île Callot au sud-est de la zone étudiée, la seconde par vent de nord-nord-ouest, dans la zone de la pointe de Primel au nord-est.

Dans la région du Trezen Vraz, au large de Roscoff (fig. 6). les lignes directrices de la topographie sont caractérisées par la direction nord-est/sud-ouest indiquée a l'est par les isobathes 50,60 et $70 \mathrm{~m}$. Mais, dans la partie sud de la carte. les isobathes s'infléchissent en direction estouest. Il en résulte un resserrement très important au nord de lîle de Batz où, en moins de 4 milles, les fonds passent de 0 à $75 \mathrm{~m}$. A cette physionomie générale se surimpose. dans la partie centrale, le promontoire constitué par la formation gravelo-sableuse du Trezen-Vraz ( la Grande Dune ") et du Bank ar Forest ("le banc de la Forêt ") qui prolonge vers le large en direction sud-est/nord-ouest, puis sud - sud-est/nord - nord-ouest, le massif dunaire qui s'appuie sur les récifs des Trépieds. La représentation du relief, sur la carte, par des isobathes, entraîne inévitablement une certaine régularisation, en ne faisant pas apparaître les inégalités inférieures à l'intervalle choisi et en arrondissant le contour des accidents de forte amplitude. La figure 6 représente deux exemples de profils. Ils illustrent la transition entre le socle, paléozoïque, et la surface structurale horizontale ou faiblement inclinćc, d’àge Lutéticn, à la surface de laquelle des cailloutis et des graviers quatemaires se mêlent à des dépôts sableux actuels. 

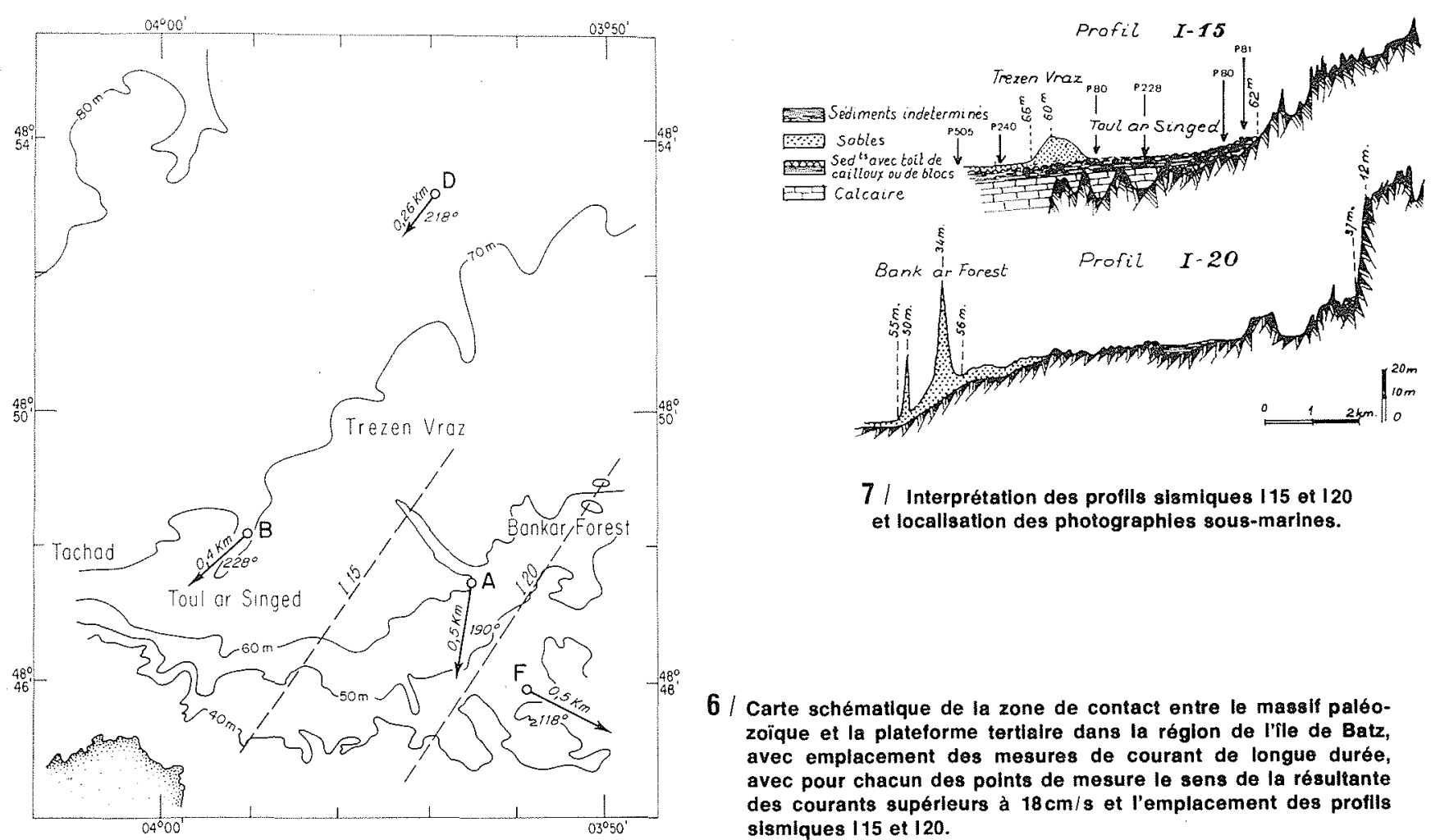

7 / Interprétation des proflls sismiques 115 et 120 et locallsation des photographies sous-marines.

6 / Carte schématique de la zone de contact entre le massif paléozoīque et la plateforme tertlaire dans la région de l'íle de Batz, avec emplacement des mesures de courant de longue durée, avec pour chacun des points de mesure le sens de la résultante des courants supérieurs à $18 \mathrm{~cm} / \mathrm{s}$ et l'emplacement des proflls sismlques 115 et 120.

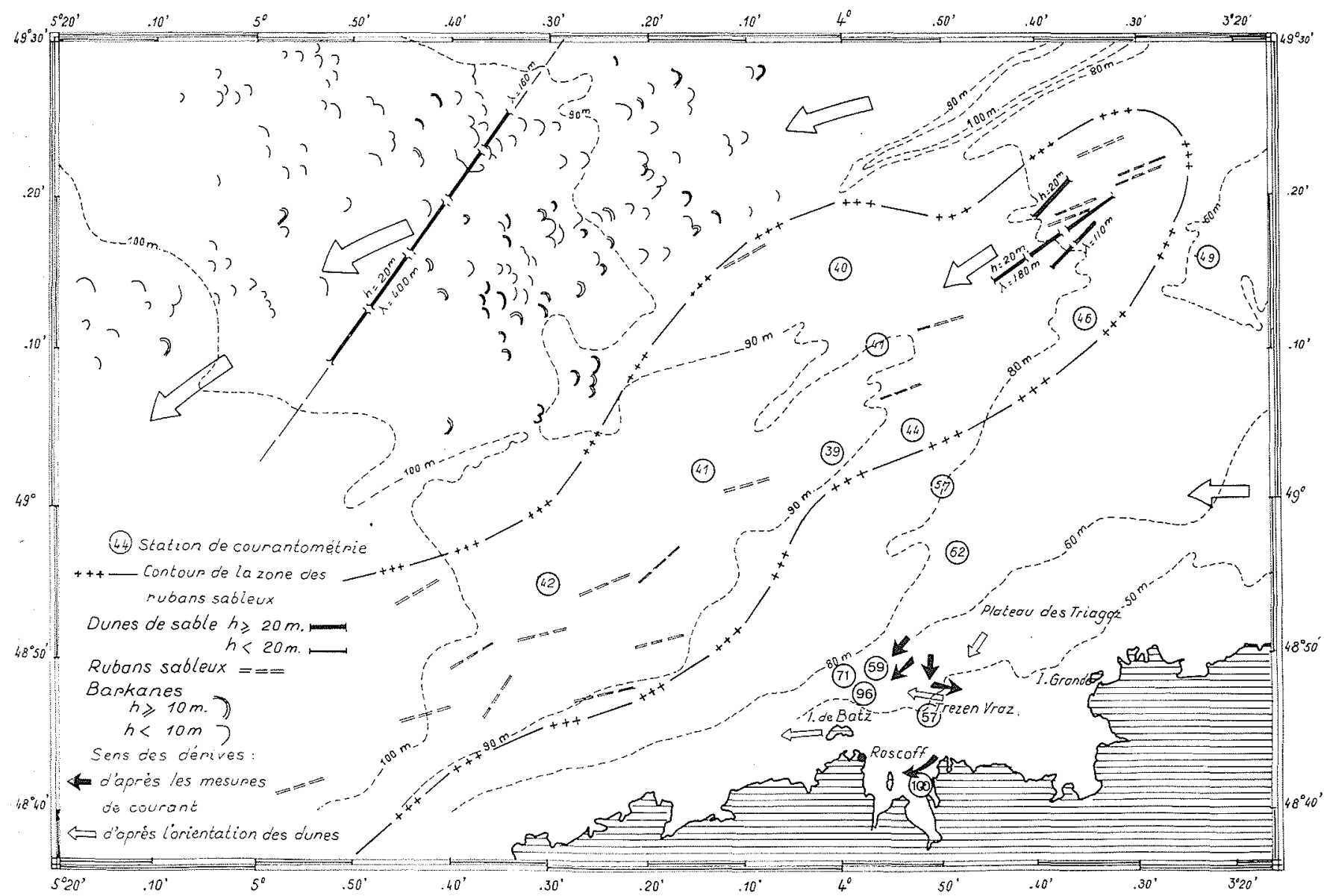

8 / Bathymétrie et types d'accumulations sédimentaires du sud de la Manche occidentale, avec, entourées d'un cercle, les valeurs maximales de la vitesse des courants pour le coefficient 95 , et le sens des transits sédimentalres. 
Profil I 15: une série de photographies sous-marines a été effectuée le long de ce profil. La photographie 89 a montré une couverture sableuse, de même que P 240 et P 505. L'épaisseur du sable. d'après les résultats de la sismique Sparker, est au maximum d'une dizaine de mètres. La photographie P 486, au-delà du profil, a montré. de nouveau, les cailloutis qui constituent donc le soubassement de l'accumulation sableuse du Trezen-Vraz.

Profil I 20: ce profil est intéressant par les deux dunes hydrauliques dont la plus importante a une amplitude de $40 \mathrm{~m}$ pour $500 \mathrm{~m}$ de longueur d'onde. Ces dunes sont orientées la face abrupte vers le sud-ouest.

Des mesures de courant de longue durée ( 8 à 15 jours) ont été effectuées en quatre stations. Nous avons calculé la vitesse critique d'érosion pour la fraction sableuse comprise entre 125 et $400 \mu$. puis la résultante des vitesses de courant de flot el cie jusant en vive-eau (coefficient 95) supérieures à cette valeur critique $(18 \mathrm{~cm} / \mathrm{s})$. Ces résultantes sont présentées en grandeur el en direction sur la figure 6 . On peut observer, aux points $D$ et $B$. une résultante dirigée sensiblement dans le sud-ouest. tandis quau point A (banc de la Forêt) elle est dirigée vers le sud, et en $F$ (les Trépieds) vers l'est. Une expérience de marquage des sables réalisée dans cette zone par Le Gorgeu et Boillot (1964) montra une dérive des sédiments vers l'est. Notre mesure confirme donc la justesse de cette observation locale. Il apparait. en effet, évident quau contact de la plateforme et du socle paléozoïque. il existera sur le fond un déséquilibre des courants. le flot l'emportant sur le jusant. en durée et en intensité: ce phénomène se fait sentir également au point $A$, mais il semble que vers le large la direction générale de la dérive des sédiments soit dirigée vers le sud-ouest. Ceci est en bon accord avec l'orientation des dunes hydrauliques présentes sur le profil I 20 (fig. 7).

Au large du Trezen Vraz. toujours au sud de la Manche Occidentale. la bathymétrie est caractérisée par des isobathes de direction nord-est/ouest - sud-ouest, tout en se resserrant vers le sud-ouest (fig. 8). Les fonds inférieurs à $90 \mathrm{~m}$ dessinent au nord-est une large dépression allongée nord-est/sud-ouest. G. Boillot (1964) a donné une carte schématique de la répartition des sédiments dans la Manche Occidentale. Il y distingue la bande cotière qui comprend notamment les accumulations sableuses au contact du socle paléozoique. une zone de cailloutis et enfin trois zones sableuses distinguées en fonction de leur teneur en Bryozoaires. Kenyon (1970), utilisant les résultats obtenus à l'aide du sonar latéral. a pu mettre en évidence l'existence au sud de la Manche Occidentale. d'une zone ou prédominent des accumulations sableuses longitudinales de faible épaisseur (5 a $10 \mathrm{~cm}$ ?) désignées sous le nom de "rubans sableux". Cette zone ne coïncide pas avec les zones distinguées par Boillot. mais chevauche la zone des sables coquilliers à Bryozoaires et la zone des cailloutis. Cette zone est bordée au nord-ouest, au nord-est, à l'est et peut-être au sud, par des zones où prédominent des rides sableuses allongées perpendiculairement au courant (Auffret et al., 1971). L'examen des photographies sousmarines a montré que les fonds de la zone des rides sableuses sont caractérisés par une grande hétérogénéité comme cela était suggéré par les dragages. Les photographies peuvent en effet montrer des rides sableuses, des fonds grossiers ensablés et ridés, des fonds caillouteux ensablés ou encore des fonds détritiques caillouteux et graveleux. Un levé bathymétrique détaillé, réalisé en
1973 par la Mission Océanographique de l'Atlantique, a rencontré un grand nombre de reliefs à pentes dissymétriques. La jonction des points hauts rencontrés a souvent permis de reconnaitre une forme de dune en croissant de type "barkhane». La pente raide située à l'intérieur du croissant est orientée vers le sud-ouest et est caractérisée par un pendage moyen de 9" (fig. 9). La figure 8 montre la localisation des formes cartographiées, elle met en évidence le groupement en triangle des formes les plus hautes dans une région relativement bien délimitée. On peut également remarquer que l'orientation d'ensemble de cette zone est sensiblement parallèle à celle de la zone des rubans sableux. Un tel groupement des champs de dunes en triangle a également été observé en mer du Nord par Mac Cave (1971).

Les courants ont été mesurés au voisinage du fond pendant douze ou vingt-quatre heures en neuf stations. La direction des courants, lors des maxima de flot et de jusant. est est-ouest dans l'ensemble de la région étudiée. Les intensités maximum indiquées sur la figure ont été extrapolées pour un coefficient de 95 , à partir de la relation empirique entre coefficient et intensité du courant établie lors de mesures de longue durée pour les stations du large (J.-L. Douville, en préparation). Un gradient d'augmentation du sud vers le nord pour ce maximum apparaît nettement; d'ouest en est la situation est plus complexe. La station 7 semble coïncider avec un minimum. Le maximum de courant augmenterait vers l'est - nord-est et vers l'ouest - sud-ouest.

\section{C) DÉBOUCHE DE LA MANCHE}

La présence de rides sous-marines sur le banc de la Chapelle, par des profondeurs de $165 \mathrm{~m}$, a été signalée dès 1958 par Cartwright et Stride; leur longueur d'onde est d'environ $850 \mathrm{~m}$ mais présente des variations. L'amplitude moyenne est de $7.6 \mathrm{~m}$, la hauteur maximum observée $12.20 \mathrm{~m}$. L'orientation des crêtes est de $110^{\circ}$, les rides étant parallèles à la direction générale du bord du plateau. Des observations complémentaires sur ces rides furent faites par l'un de nous (L. Berthois. 1959) (fig. 10) qui montra en outre que leurs pentes sont accidentées de petites rides secondaires ayant 30 à $40 \mathrm{~m}$ de longueur d'onde et 2.5 à $3 \mathrm{~m}$ de hauteur (fig. 11 ). Ces rides sont symétriques ou orientées face abrupte au nord - nord-est tandis que la face abrupte des petites rides qui les accidentent semblent orientées vers le sud-ouest.

Mais, de façon générale, il apparaît (Stride, 1963) que la tendance générale des rides s'accorde avec un mouvement vers le sud-ouest, c'est-à-dire vers la rupture de pente du plateau continental au voisinage de laquelle une tendance contraire peut être observée. Les sédiments prélevés dans la zone du banc de la Chapelle sont assez mal triés et ont des médianes comprises entre 240 et $510 \mu$. Deux populations peuvent être observées (fig. 4), la première de 12 à $1 \mathrm{~mm}$, la seconde, de $1 \mathrm{~mm}$ à $89 \mu$, représente environ $75 \%$ du sédiment total. Au moment de la rédaction de cet article, l'auteur ne disposait d'aucune donnée sur l'intensité des courants du voisinage du fond dans ce secteur. Se fondant sur les distributions granulométriques observées, il proposa alors l'idée que ces grands corps sableux s'étaient édifiés lors de bas niveau marin du quaternaire et qu'ils étaient actuellement stabilisés dans leur grand trait, bien qu'occasionnellement ils 


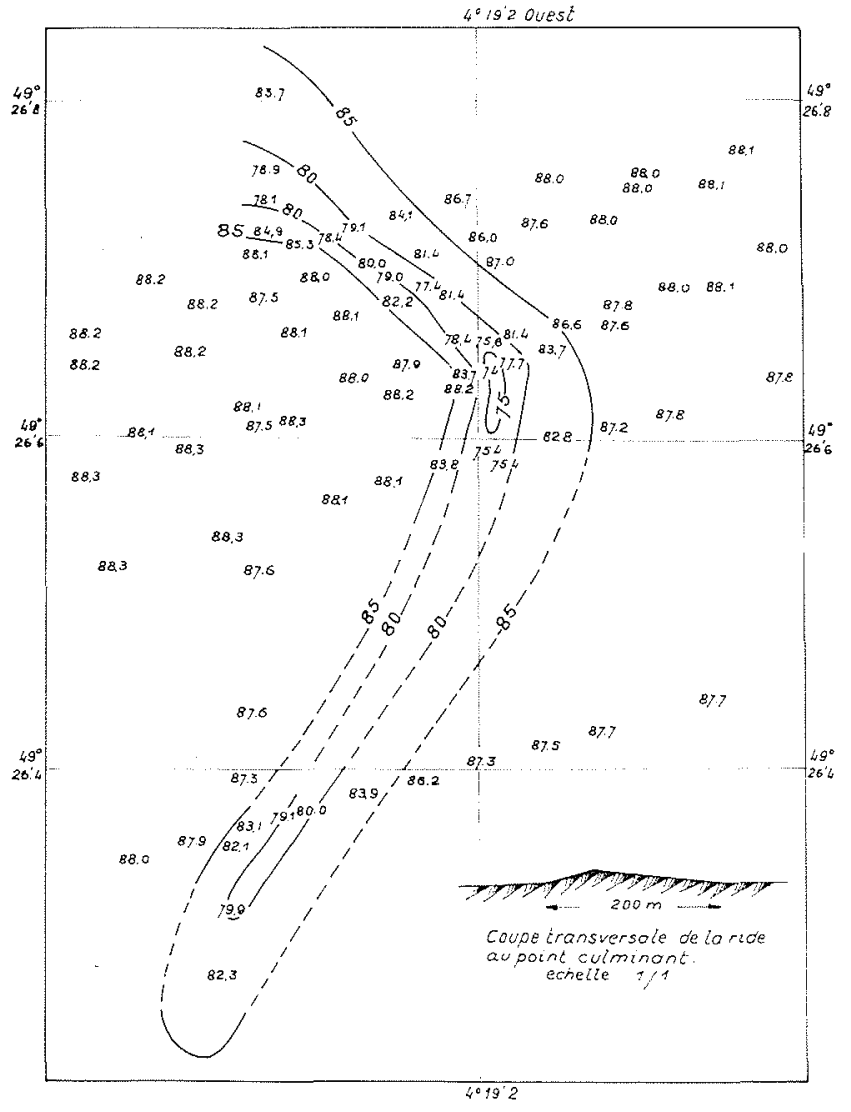

9 / Levé à grande échelle d'une barkhane dressé par le Service Hydrographique et Océanographique de la Marine.

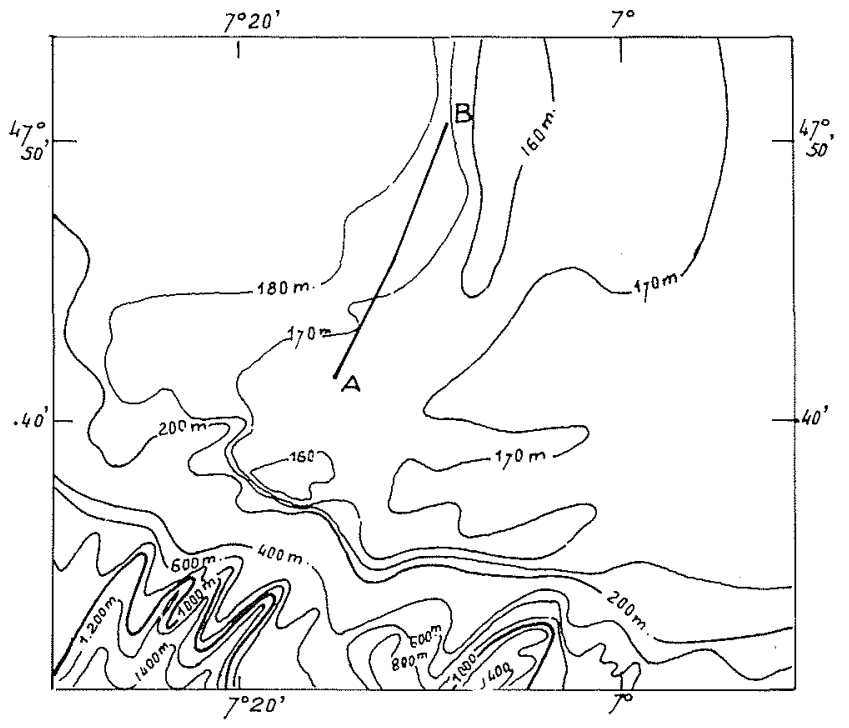

10 / Carte schématique du banc de la Chapelle, avec localisation du profil bathymétrique AB.

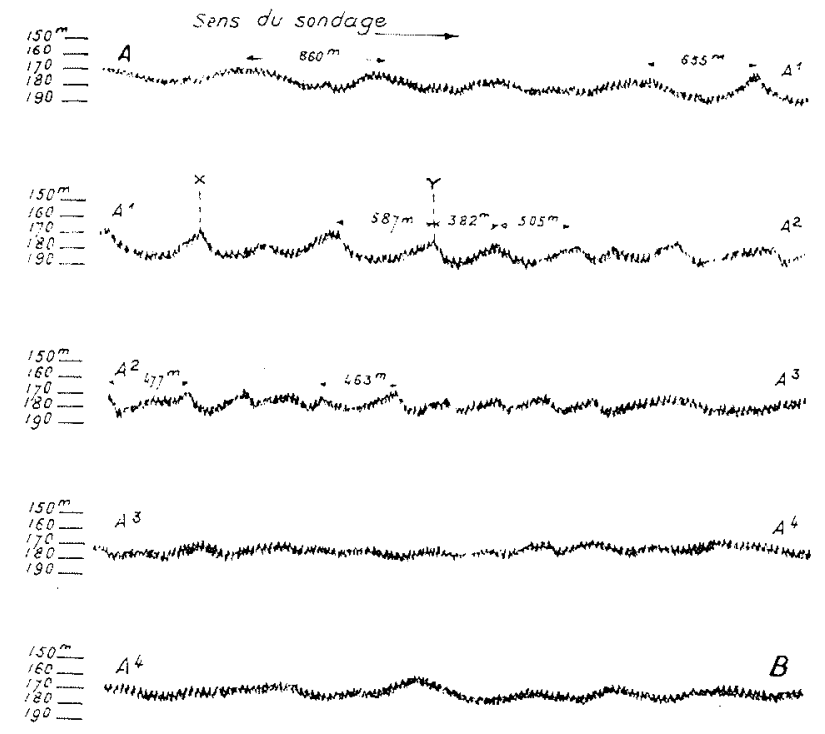

11 / Profil bathymétrique des dunes du banc de la Chapelle selon le profil AB (figure 10). 


\begin{tabular}{|c|c|c|c|c|c|c|c|c|c|c|}
\hline \multicolumn{11}{|c|}{ Tableau 1} \\
\hline & $\begin{array}{c}\text { Md } \\
\mathrm{cm} \times 10^{-4}\end{array}$ & $\begin{array}{c}D * \\
\mathrm{~cm} \times 10^{-4} \\
\end{array}$ & $\mathrm{R}_{*}$ & $\begin{array}{l}U_{*} \\
\mathrm{~cm} / \mathrm{s} \\
\end{array}$ & $\begin{array}{l}V_{i 0} \\
\mathrm{~cm} / \mathrm{s} \\
\end{array}$ & $\begin{array}{c}V_{H} \\
\mathrm{~cm} / \mathrm{s} \\
\end{array}$ & $\begin{array}{c}V_{T} \\
\mathrm{~cm} / \mathrm{s} \\
\end{array}$ & $\begin{array}{l}U_{* T} \\
\mathrm{~cm} / \mathrm{s} \\
\end{array}$ & $\begin{array}{l}\Delta U_{*} \\
\mathrm{~cm} / \mathrm{s} \\
\end{array}$ & $\begin{array}{c}J_{M} \\
\mathrm{~kg} / \mathrm{m} / \mathrm{s} \\
\end{array}$ \\
\hline Baie de Morlaix ........ & & & & & $>25$ & $<50$ & $\simeq 75$ & & & $?$ \\
\hline Pierre Noire (point $Y$ ) $\ldots$ & 155 & 3,4 & 1.4 & 1,1 & 100 & 200 & 300 & 17 & 16 & $?$ \\
\hline Les Trépieds (point $F$ ) ... & 400 & 9.2 & 5 & 1,5 & 57 & 82 & 139 & 7.9 & 6,4 & 27.7 \\
\hline Point $\mathrm{Xl}$ & 550 & 12.6 & 7 & 1.5 & 62 & 50 & 112 & 6.3 & 4.8 & 35.7 \\
\hline Point $x \ldots \ldots \ldots \ldots$ & 500 & 11,5 & 6.5 & 1.5 & 57 & 48 & 105 & 5.9 & 4,4 & 27.7 \\
\hline Point IV $\ldots . . . \ldots \ldots$ & 500 & 11.5 & 6.5 & 1.5 & 44 & 46 & 90 & 5,1 & 3.6 & 0,65 \\
\hline Point $V \ldots \ldots \ldots \ldots$ & 500 & 11.5 & 6.5 & 1.5 & 41 & 46 & 87 & 4.9 & 3.4 & 0.35 \\
\hline Point VI $\ldots \ldots \ldots \ldots$ & 400 & 9,2 & 5 & 1.5 & 40 & 46 & 86 & 4.8 & 3,3 & 0.32 \\
\hline \multicolumn{11}{|c|}{ LÉGENDES } \\
\hline \multicolumn{5}{|c|}{$\begin{array}{l}\text { Md médiane; } \\
D . \quad \text { diamètre sédimentologique; } \\
\mathrm{R} \text {. nombre de Reynolds étoilé; } \\
U . . \text { vitesse de frottement correspondant ail début de } \\
\text { rentrainement des matériaux; } \\
V_{3} \text { vitesse mesurée au courantographe } 70 \mathrm{~cm} \text { au-dessus du } \\
\text { fond marin; }\end{array}$} & \multicolumn{6}{|c|}{$\begin{array}{l}\text { vitesse théorique sur le fond induit par les houles de } \\
\text { secteur } \mathrm{O}-\mathrm{N}-\mathrm{O} .\left(T_{n}=12 \mathrm{~s} \text { et } \lambda_{n}=100 \mathrm{~m}, 2 a_{n}=6 \mathrm{~m}\right) \\
\text { vitesse résultant de laddition des deux composantes; } \\
\text { vitesse de frottement correspondante; } \\
\text { ecart cntre } U \cdot \text { et } U \cdot r ; \\
\text { débit solide maximum théorique. }\end{array}$} \\
\hline
\end{tabular}

puissent être l'objet de "remaniement " d'amplitude difficile à estimer. Une mesure des courants profonds de cette région a été effectuée, en 1970, par le Centre Océanologique de Bretagne (A. Cavanié et J.-L. Hyacinthe, sous presse). Des vitesses maximales de $50 \mathrm{~cm} / \mathrm{s}$ au-dessus du fond ont été observées, ces vitesses se produisant lorsque le courant a une direction perpendiculaire à la rupture de pente du plateau continental et à la crête des rides.

\section{Hypothèses sur la genèse des formes sédimentaires}

Les observations précédentes suggèrent qu'il existe une hiérarchie des formes d'accumulation sédimentaires. depuis les rides de courant assymétriques de $n \times 10 \mathrm{~cm}$ de longueur d'onde cobservables sur les photographies sousmarines) aux grands bancs de $n \times 10 \mathrm{~km}$ de longueur. allongés le plus souvent selon le plus grand axe de l'ellipse des courants de marée. Une forme intermédiaire au moins existe. Il s'agit des grandes dunes ou barkhanes, hautes de 10 à $20 \mathrm{~m}$ et d'une longueur d'onde de $n \times 100 \mathrm{~m}$, qui reposent sur un fond plat ou qui festonnent le flanc des grands corps sableux. Nous avons noté que ces trois formes pouvaient localement être présentées simultanément. Existe-t-il une relation génétique entre elles? Ou correspondent-elles à des processus sédimentaires indépendants, de niveaux d'énergie différents? En d'autres termes. à la place de l'hypothèse précédente. peut-on imaginer que les corps sableux résultent d'un processus d'accrétion (continuel ou occasionnel) des dunes ? La réponse a ces questions est rendue difficile par le fait que ces formes se sont constituées au cours dune histoire géologique pendant laquelle les fluctuations glacio-eustatiques du niveau marin ont été considérables (de l'ordre d'une centaine de mètres). Les conditions hydrauliques actuelles n'ont donc peut-être rien à voir avec celles ayant prévalu au cours de leur genèse. Il n'en est pas moins vrai que les processus sédimentaires sont actuellement actifs. Les grands traits morphologiques actuels de ces accumulations sont donc peut-être le reflet d'un équilibre dynamique stable, érosion et sédimentation se compensant localement (Hinsberger. 1970).

\section{Essai de synthèse}

Nous disposons donc de données relativement précises sur la morphologie. la pétrographie et les conditions dynamiques au voisinage des accumulations sableuses en Manche et en mer Celtique (tableau 1). En ce qui concerne l'agitation près du fond due à la houle. nous avons évalué ses valeurs dans la région de Roscoff pour les conditions suivantes:

- Houle de secteur ouest-nord-ovest et de période $12 \mathrm{~s}$, de longueur d'onde égale à $100 \mathrm{~m}$. et d'amplitude (2 a) égale à $6 \mathrm{~m}$. Le calcul montre que ces valeurs décroissent 
vers le large de $2 \mathrm{~m} / \mathrm{s}$ à la Pierre Noire à $46 \mathrm{~cm} / \mathrm{s}$ au niveau de l'isobathe $80 \mathrm{~m}$. D’après le modèle DS A 5 de la Météorologie Nationale, une telle intensité ne se rencontrerait au cours d'une année que sept fois environ durant six heures (A. Cavanié, ce volume). D’après le même auteur, par $100 \mathrm{~m}$ de fond des vitesses de $35 \mathrm{~cm} / \mathrm{s}$ induites par la houle seraient observables dans $1.5 \%$ des cas, c'est-à-dire l'équivalent de 4.8 jours par an. D'après Draper (1967), par $90 \mathrm{~m}$ de profondeur. la vitesse de $33 \mathrm{~cm} / \mathrm{s}$ induite par la houle serait dépassée au large de la Cornouaille pendant l'équivalent d'une durée de 1.8 jour annuellement, et 3.6 journées par an en mer Celtique. Les ordres de grandeur de ces évaluations sont donc comparables. Dans une publication récente (Ewing, 1973). l'auteur précisant le modèle spectral de la houle, parvenait à la conclusion que les vitesses estimées par Hadley (1964) et Draper (1967) devaient être doublées pour des conditions de vent identiques. Par ailleurs. le même auteur souligne le fait que les sinuosités de la rupture de pente du plateau continental doivent occasionner une réfraction des grandes longueurs d'onde et, par conséquent, une concentration de l'énergie sur les bancs tels que celui de la Chapelle.

Les vitesses de frottement théoriques correspondant aux valeurs précédentes des courants de marée et de houle ont été estimées en utilisant les formules de Sternberg (1968), les valeurs critiques pour le déplacement des sédiments d'après les abaques de Bonnefille (1963) et les résultats de nos analyses granulométriques. La différence entre ces deux valeurs peut être prise comme indicateur de l'intensité des processus sédimentaires dans les différentes zones. Lexamen du tableau conduit aux conclusions suivantes C'est dans les milieux frontolittoraux, tels ceux de la Pierre Noire, que l'énergie disponible pour le transport sédimentaire est la plus importante. C'est dans cette zone que la médiane des sédiments est la plus faible, tandis qu'au large où les médianes de la fraction sableuse sont plus élevées, lénergie disponible est moindre. Nous devons signaler en outre qu'il existe dans cette zone une nappe de cailloutis et de gravier "reliques" dont nous n'avons pas tenu compte pour ce calcul.

Lévaluation de la force tractrice effective et de la force tractrice critique permet, d'après Sternberg (1972), de calculer le transport massique effectif. Le calcul fait apparaître une diminution très nette de l'intensité du transport entre le point $X$ et le point IV. puisque le débit massique maximum théorique varie de $27.7 \mathrm{~kg} / \mathrm{m} / \mathrm{s}$ à $0.65 \mathrm{~kg} / \mathrm{m} / \mathrm{s}$. Il est remarquable de constater que cette différence ne correspond quà une relativement faible diminution du maximum des vitesses de $57 \mathrm{~cm} / \mathrm{s}$ à $44 \mathrm{~cm} / \mathrm{s}$, mais qu'en fait elle correspond de façon précise à la limite entre la zone des rubans sableux et celle des accumulations sableuses littorales. Des observations réalisées sur une accumulation sableuse de la mer dillande ont permis d'estimer l'importance du débit solide. Celui-ci, moyenné sur une période de 42 jours, atteindrait localement $0.7 \mathrm{~m} 3 / \mathrm{m} / \mathrm{j}$ ou, si l'on adopte une densité de 2. 1,4 t/m/j (Jones et al.. 1965).

On observe donc en Manche et en mer Celtique une hiérarchie de formes d'accumulations sédimentaires, allant des grands corps sableux (exemple de la Bassurelle) aux petites rides de 30 a $50 \mathrm{~cm}$ de longueur d'onde qui accidentent la surface des grandes dunes. La notion d'activité, pour de tels phénomènes, n'a de sens qu'en fonction de l'échelle spatiale et temporelle à laquelle on se situe. Ainsi localement et à certain moment, ainsi que l'ont montré les mesures effectuées au nord-est de la dune des Trépieds, le transit sableux peut avoir une direction différente de la dérive régionale (dirigée vers le sud-ouest).

La géométrie et la dynamique des grands corps sableux (la Bassurelle, la Chapelle) doit être étudiée à l'échelle du quaternaire $\left(10^{\circ}\right.$ ans), leur évolution à l'échelle de l'année ou de la décade est probablement négligeable. Les grandes dunes sableuses qui les accidentent, ou qui occupent les fonds plats de la Manche, évoluent sans doute d'une part de manière continue mais sans doute peu sensible, en relation avec les composantes résiduelles de la marée, d'autre part de façon brutale. lors des grandes perturbations atmosphériques coincidant avec de fortes marées. Leur évolution doit sans doute pouvoir se mesurer à l'échelle de l'année ou de la dizaine d'année, si une localisation suffisamment précise est disponible. Des forages britanniques et hollandais, dans la région Pas-de-Calais (Dr. Cloet, communication personnelle), ont montré qu'en fait les dunes hydrauliques y étaient de deux types, indifférentiables par la seule bathymétrie. Le premier type comprend des dunes constituées sur toute leur épaisseur de sable homogène. Dans le second type, la granulométrie varie : grossières à la base (lit de galets), puis sableuses, ces dunes se terminent parfois au sommet par des lits vaseux. Ce type de dune s'est vraisemblablement mis en place à une époque où le régime des courants dans leur zone d'occurrence était plus actif que de nos jours. A la suite d'une décroissance de l'énergie disponible, leur forme a été fossilisée. On conçoit aisément l'importance de cette distinction du point de vue scientifique et du point de vue pratique pour la détermination des tracés de chenaux empruntés par les navires. Mais, faute de tels forages, nous ne pouvons préciser ce point dans la région étudiée. Les petites rides sableuses oscillent sans doute journellement et progressent dans le sens du courant résiduel. Leur évolution doit donc, selon lintensité du courant, pouvoir se suivre depuis l'échelle journalière jusqu'à l'échelle saisonnière. Il est probable qu'en certains points elles peuvent s'effacer pour de courtes périodes quand le nombre de Froude est supérieur à 1 pour se reformer quand le courant décroît.

En ce qui concerne l'orientation de ces différentes formes par rapport à l'éllipse des courants de marée, et à la direction du transport résiduel, aucune conclusion ne peut être avancée en l'absence de données suffisantes. On peut seulement faire remarquer que les grands corps sableux du nord de la Manche Orientale sont allongés suivant le grand axe de l'ellipse et que la crête des grandes rides est légèrement oblique par rapport à cette direction. Les grandes dunes à crête rectiligne ou les barkhanes ont, au contraire, leur crête perpendiculaire au grand axe de l'ellipse. En tout état de cause, la forme de ces accumulations doit être également tributaire, pour des conditions hydrodynamiques données, du volume du matériel disponible pour le transport; mais, en retour, la géométrie des accumulations a probablement une influence directe sur l'hydrodynamisme des écoulements.

\section{Conclusion}

La localisation des grands corps sableux semble avoir été déterminée par des conditions géologiques indépendantes des conditions océanographiques actuelles, la localisation des grandes dunes est le produit des actions marines 


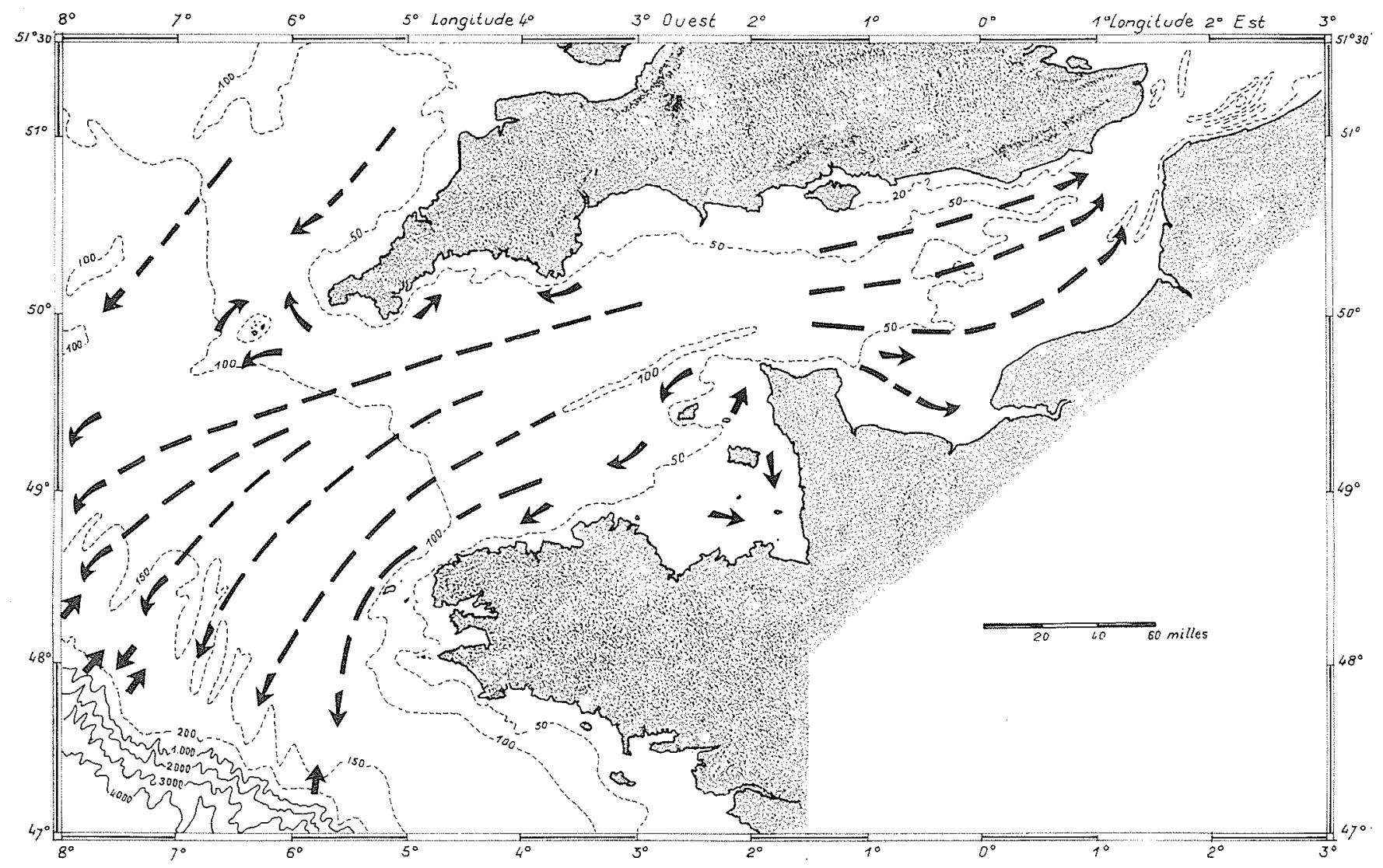

12 / Sens des transits sableux en Manche et en mer Celtique d'après Belderson, Kenyon et Strlde.

des 16000 dernières années qui redistribuent le matériel précédemment déposé dans d'autres conditions, la tendance de ce mouvement semble être une accumulation de ce matériel vers la rupture de pente du plateau continental.

La cause d'un tel mouvement n'apparaît pas clairement. elle pourrait être liée à une compensation, par un courant profond, de l'excédent massique du flux d'eau atlantique de surface qui caractérise le régime hydrologique de la Manche Occidentale, particulièrement en hiver (Dietrich. 1950). Une autre explication pourrait être recherchée dans laccentuation de l'amplitude des houles lors de la conjugaison des courants de jusant et d'une tempête de secteur ouest. L'accentuation de l'amplitude du mouvement dû̀ la houle pourrait favoriser l'intensité des remaniements sédimentaires pendant le jusant et expliquer l'assymétrie observée (A. Cavanié, communication personnelle). Cette hypothèse n'explique pourtant pas, à elle seule, les phénomènes observés et notamment l'inversion du sens du transport vers le golfe normano-breton et en Manche Orientale (fig. 12)

\section{Remerciements}

Il nous est particulièrement agréable de remercier $M$. l'ingénieur en Chef de l'Armement Schrumpf, du Service Hydrographique et Océanographique de la Marine, M. Cabioch, Sous-Directeur de ta Station Biologique de Roscoff,
M. Laubier. Chef du Département Scientifique du C.O.B.. et $M$. Cavanié, responsable de l'équipe d'Océanographie Physique, pour leur aide el leur coopération.

\section{Bibliographie}

AlLEV (J.R.L.). - "Current Ripples". Amsterdam, NorthHollond. Pub. Co.. 433 pp. (1968).

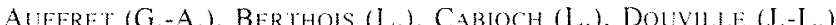
- Contribution à létude et à la cartographie des fonds sédimentaires au large de Roscoff. Actes du colloque sur la Manche. janvier 1971. Paris. Mémoires du B.R.G.M., 79 : 293-302.

Auffret (G.-A.), Berthols (L.), CABIOCH (L.), DOUville (J.-L.), KEVYOV (N.-H.). - Aperçu sur les structures sédimentologiques à grande et petite échelle et les processus sédimentaires actuels au sud de la Manche Occidentale. Ann. Inst. Océ(110). tome XLVIII. fasc. 2 (1972).

AufFret (G.-A.). DouvILLE (J.-L.). - Observations sur la dynamique des sables de la Pierre Noire (baie de Morlaix. Finistère, France) Bull. B.R.G.M., 2" S.. Sect. IV. n" | (1974).

Aufrete (G.-A.). Douville (J.-L.). - Faciès sédimentaires actuels à la périphérie ouest et nord du craton armoricain. relations avec les processus dynamiques. IX" Congrès International de Sédimentologie. Nice 1975 (sous presse).

Beldorsoy (R.H.). Kevyon (N.H.). STRIDE: (A.H.). - Holocene sediments on the continental shelf west of the British Isles. The geology of the East Atlantic Continental Vargin. Edited by F.M. Delam (1971). Institute of Geological Sciences Report n" 70/14. pp. 157-170.

BHERTHOS (L.). - Remarques sur les rides sous-marines. Rev. des Wur. de l'ISTPM, tome 23, pp. 225- 239 (1959). 
BERTHOLS (L.), BREYOT (R.), AUFFRET (G.A.), DU BUIT (M.-H.) - La sédimentation dans la région de la Bassurelle (Manche Orientale). Cah. Centre Rech. et Ét. océtunogr. VIII. fasc. 3 (1969).

Bontror (G.). - Géologie de la Manche Occidentale. Ann. In.st. océanog. tome XLII (1). 2209 (1964)

BONEFll. l. (R.). - Etude de synthèse des lois de début d'entraînement des sédiments sous l'action d'un courant en régime continu. Bull. Direction EI. Re'C. E.D.F., n" 5, p. 67 (1963).

$\mathrm{CABHOCH}(\mathrm{L}$.$) . - Contribution a la connaissance des peuplements$ benthiques de la Manche Occidentale. Cah. Biol. mar.. 9 (5) : 493-720 (1968)

Car IRIGH (D. E.) and Stride (A. H.). - Large sand wawes near the edge of the continental shelf. Namure. 181-41 (1958).

Cavane (A.). - Application à la zone au large du Finistere dune méthode destimation statistique des courants de houle au voisinage du fond. Actes de la Session de la Société Hydrotechnique de France des 19 et 20 juin 1975 (sous presse).

Carave (A.). HYACINTHE (J.-L.). - Étude des courants et de la maré a la limite du plateau continental d apres les mesures effectuées pendant la campagne "Golfe de Gascogne 1970\%. Publications techniques du C.N.E.X.O. (sous presse).

DAVGEARD (L.). - Observations de géologie sous-marine et d'océanographie relatives à la Manche. Alm. Inst. Ocüumog. Paris. N.S. 1. VI. fasc. 1. 296 p. (1928).

DIETRICH (G.). - Die anomale Jahresschuankung des Wärmeinhalts in Englischen Kanal, ihre Ursachen, und Auswirkungen. Deut. Hydrograph. 2.. 3. 184-201 (1950).

Dotvilfe: (J.-L.). - Estimation de l'hydrodynamisme. due à la houle au niveau du fond, en baie de Morlaix. Bull. de IU.O.F. vol. VI, Bill. 4, pp. 18 a 23 (1974).

DrAPER (L.). - Wave activity at the sea bed around northwestern Europe Marine Geology' 5, 133-140 (1967).

Ewsis (J.A.). - Wave induced bottom currents on the outer shelf. Marine Geology. 15: M 31-M 35 (1973).
HADLEY (M. L.). - Wave induced bottom currents in the Celtic Sea. Marine Geology: 2 : 164-167 (1964)

Hivsberger (F.). - "L'Iroise et les abords d'Ouessant et de Sein *. Université de Caen. Faculté des Lètres et Sciences Humaines. 309 p. (1970).

Jovfes (N.S.), KAIN (J. M.) and STRIDE (A. H.). - The movement of sand waves on Warts Bank, Isle of Man. Marine Geolog. 329-336 (1965)

Kevyoy (N. H.). - Sand ribbons of european tidal seas. Marine Geology, $9: 25: 39$ (1970)

KeNYoN (N. H.). STride (A. H.). - The tide-swept continental shelf sediments between the Shetland isles and France. Sedimentology, $14:$ 159-173 (1970).

KEVYON (N. H.). - The origin of some transverse sand patches in the Celtic Sea. Geol. Mag.. pp. 389-394 (1970).

Le: Gorgeu (V.-P.). Bolllot (G.). - La migration de sables marqués au large de Roscoff (Finistère). C.R. Acad. Sc.. Paris, l. 259 , pp. $4082-4085$ (1964).

Mc CAVE (I. N). - Sand waves in the North Sea off the coast of Holland. Marine Geology, v. 10, pp. 149-227 (1971).

Pruvot (G.). - Essai sur les fonds, et la faune de la Manche Occidentale (1897). Arch. Zool. Exper. Gès. Paris, 3" série, t. V.. pp. 511-614.

SUITH (J. D.). - Geomorphology of a sand ridge (1969). Joum Geology. v. 77, pp. 39-55.

STERVBERG (R. W.). - Friction factors in tidal channels with differing bed roughness (1968). Marine Geology, $6: 243-260$.

STIRNBBERG (R. W). - Predicting initial motion and bedload transport in shelf sediment transport (1972). Dowden, Hutchinson \& Ross. Lis. Edit.. pp. 61-82.

STRIi)E (A. H.). - Shape and size trends for sand waves in a depositional zone of the North Sea (1970). Geol. Mag.. pp. 469-477.

Thoulet (J.). - Carte lithologique sous-marine des cotes de France (1922). Chalamel. Edit. Paris.

\title{
Discussion
}

\author{
Président: M. le Professeur LACOMBE
}

M. le Président remercie M. Auffret pour son importante contribution à la description de certains caracteres des fonds sur la plateforme continentale armoricaine.

Il ouvre ensuite la discussion en espérant que celle-ci apportera des informations complémentaires sur certaines observations curieuses concernant, notamment, le sens des courants par rapport au cheminement des sédiments.

A la lumière des observations faites notamment à l'occasion de l'étude du port d'Antifer, M. Couprie présente son point de vue dans une intéressante intervention qu'il a résumée comme suit :

Pour expliquer les sens différents du transit littoral de part et d'autre du Cotentin, on peut invoquer la nature très différente des houles de part et d'autre de la presqu'île: à l'ouest les houles longues de l'Atlantique pénètrent dans la Manche tandis qu'à l'est. les études d'Antifer montrent que les composantes à longue période n'existent pas; ce n'est qu'une houle de vent très vite levée et très sensible au courant de marée.

L'action sur les fonds est d'ailleurs différente du fait des profondeurs relatives variables d'une région à une autre.

L interaction courant-houle reste encore à mieux préciser.

M. ZIMMERMAvV (Sté Technip) pose les deux questions suivantes:

1" Le point de divergence des transports de sédiments correspond grossièrement en Manche avec celui d'amplitude minimum des marées. Pensez-vous que ces deux phénomènes soient liés?

2" Comment a été déterminée la vitesse $18-20 \mathrm{~cm} / \mathrm{s}$ considérée conme critique pour déclencher un transport de sédiment?
Sur le premier point, M. le Président et $M$. Aufrre t observent que c'est dans cette région de la Manche que les courants de marée atteignent leur maximum et on $y$ a mis en évidence, non pas des rubans sableux, mais des formes d'érosion du fond, qui poumaient être liées aux courants de marée (sillons de $1 \mathrm{~m}$ de profondeur). La provenance des matériaux est relatıvement compliquée à déterminer car il s'agit, en général, de sédiments anciens mis en place par les rivières et les fleuves à l'époque où les fonds de la Manche étaient émergés.

En ce qui concerne la deuxième question posée par M. ZIMMERMANN, M. DOUVILLE apporte les informations ci-après

L'analyse de la distribution granulométrique des sédiments prélevés a permis de mettre en évidence trois populations granulométriques différentes; la détermination de la médiane de chacune de ces populations permet le calcul de la vitesse critique susceptible d'en assurer la première mise en suspension d'après l'abaque de M. Bonnefille. La vitesse de $18 \mathrm{~cm} / \mathrm{s}$ correspond à la fraction la plus fine des sédiments prélevés.

Sur une question de $\mathrm{M}$. le Président, M. AufFret précise que les vitesses de l'eau étaient mesurées à $0,75 \mathrm{~m}$ du fond environ. Pour en déduire la vitesse au fond, nous avons utilisé, dit-il, les recherches expérimentales de Sternberg qui ont abouti à des abaques permettant de déterminer la " vitesse de frottement " à partir de la vitesse mesurée à $1 \mathrm{~m}$ du fond (extrapolation suivant une courbe logarithmique)

Cet auteur a mis au point une méthode pour déterminer, en fonction de la vitesse à $1 \mathrm{~m}$ du fond et de la granulométrie d'un 
fond sableux, le débit massique maximum de sédiments sur le fond. Dans notre cas, nous avons ainsi calculé des débits massiques de l'ordre de $10 \mathrm{~kg} / \mathrm{m} / \mathrm{s}$ au moment des maxima de courant.

Des mesures effectuées par des chercheurs anglais, à partir de la vitesse de translation des crêtes de dunes, ont abouti à des débits massiques de l'ordre de $0.7 \mathrm{~m}^{3}$ par mètre et par jour environ dans des conditions comparables aux nôtres (vitesse maximum du courant : $0,50 \mathrm{~m} / \mathrm{s}$ ).

M. LePETIT (L.N.H., Chatou) signale que, lors de mesures par traceurs radioactifs effectuées pour l'étude de l'avant port de Dunkerque, on a abouti à des ordres de grandeur voisins de ceux cités par M. Auffret. Pour des courants de marée variant de 0 à $1 \mathrm{~m} / \mathrm{s}$, le débit solide a été estimé à $1 \mathrm{~m}^{3}$ environ par mètre et par jour.

Sur une question de M. le Président, M. Auffret donne quelques informations sur les petites rides fugitives qui se forment sur les grandes rides. Sur des photographies ponctuelles nous avons observé, dit-il, un renversement de la symétrie des dunes selon que la photographie était prise à la fin du flot ou à la fin du jusant.

M. le Président clôt la discussion et donne la parole à $\mathrm{M}$. CouPRIE pour l'exposé de sa communication.

\section{Abstract \\ Observations on sand dunes on the continental shelf}

\section{I. - Introduction}

The growing economic interest shown in the shallower regions of the continental shelf explains the present need to know more about the contribution processes of the various sedimentary deposits.

\section{II. - Morphological forms of sedimentary accumulation}

The regions studied (Figure 1) permitted investigation of the various possible forms of accumulation, from the large sand bodies (Straits of Dover; La Chapelle bank) to the sand ribbons present at the south-western end of the Channel.

The large sand bodies of the Bassurelle running parallel to the principal tidal currents are flanked by large sand dunes whose crests are approximately parallel to the current. The ripple-marks seen on the surface of the dunes are due to present-day movement, as proved by underwater photographs. However. a colony of Lanice Conchilega of uniform age and short life cycle (2-21/2 years) points to a period of stability following the probable occurence of a meteorological disturbance

In the south-western region of the Channel, measurement of tidal currents shows that currents are predominantly south-westerly, except to the south of the Bank ar Forest where a south-easterly direction has been observed.

Two sectors (I 15 and 120 ) illustrate the transition between the Paleozoic substratum and the Lutetian sedimentary platform covered by pebble deposits and sandbanks, e.g. Trezen Vraz and Bank ar Forest (Figure 6). Figure 9 gives an example of a "Barkhan"-type crescent dune found in the southern part of the western Channel, to the north of the sand ribbon zone. The dunes on La Chapelle bank at a depth of $165 \mathrm{~m}$ (Figures 10 and 11) appear to be due to movement towards the south-west. The large sand bodies themselves are probably now quite stable.

\section{Conclusion}

Measurement of tidal currents and theoretical studies on the effect of waves in a number of sectors have provided an estimate (Table 1) of maximum probable sediment transport values (Bonneville, Sternberg). These are quite high, and range from 27.7 to $0.65 \mathrm{~kg} / \mathrm{m} / \mathrm{s}$. It is clear that small differences in maximum current velocity give rise to large differences in the mass transport value. 